\title{
Diamagnetic levitation enhances growth of liquid bacterial cultures by increasing oxygen availability
}

\author{
Camelia E. Dijkstra ${ }^{1}$, Oliver J. Larkin¹, Paul Anthony ${ }^{1}$, Michael \\ R. Davey ${ }^{1}$, Laurence Eaves ${ }^{2}$, Catherine E. D. Rees ${ }^{1}$ and Richard \\ J. A. Hill ${ }^{2, *}$ \\ ${ }^{1}$ School of Biosciences, University of Nottingham, Sutton Bonington Campus, Loughborough \\ LE12 5RD, UK \\ ${ }^{2}$ School of Physics and Astronomy, University of Nottingham, Nottingham NG7 2RD, UK
}

\begin{abstract}
Diamagnetic levitation is a technique that uses a strong, spatially varying magnetic field to reproduce aspects of weightlessness, on the Earth. We used a superconducting magnet to levitate growing bacterial cultures for up to $18 \mathrm{~h}$, to determine the effect of diamagnetic levitation on all phases of the bacterial growth cycle. We find that diamagnetic levitation increases the rate of population growth in a liquid culture and reduces the sedimentation rate of the cells. Further experiments and microarray gene analysis show that the increase in growth rate is owing to enhanced oxygen availability. We also demonstrate that the magnetic field that levitates the cells also induces convective stirring in the liquid. We present a simple theoretical model, showing how the paramagnetic force on dissolved oxygen can cause convection during the aerobic phases of bacterial growth. We propose that this convection enhances oxygen availability by transporting oxygen around the liquid culture. Since this process results from the strong magnetic field, it is not present in other weightless environments, e.g. in Earth orbit. Hence, these results are of significance and timely to researchers considering the use of diamagnetic levitation to explore effects of weightlessness on living organisms and on physical phenomena.
\end{abstract}

Keywords: diamagnetic levitation; bacterial growth; convection; sedimentation; simulated microgravity; weightlessness

\section{INTRODUCTION}

It is important to understand how weightlessness influences bacterial behaviour, not only for the health of astronauts, but also for the long-term future of space exploration [1]. Earth-based techniques can simulate aspects of a microgravity environment, but are either time-limited to a few seconds or minutes (drop towers, parabolic flights and sounding rockets), or use rotation to time-average the gravity vector to zero, which can introduce artefacts owing to the rotating reference frame (clinostats, random positioning machine; [2]).

Here, we use the diamagnetic force induced by a strong, spatially varying magnetic field to balance the force of gravity [3-7]. Just as the centrifugal force balances the gravitational force on an orbiting spacecraft, the diamagnetic force opposes the force of gravity on a levitating object. The potential of diamagnetic levitation as a laboratory-based tool to investigate the effects of weightlessness on living organisms was first demonstrated by Valles et al. [5], who studied levitating frog embryos, and by Berry \& Geim [4] who levitated a live

*Author for correspondence (richard.hill@nottingham.ac.uk).

Electronic supplementary material is available at http://dx.doi.org/ 10.1098/rsif.2010.0294 or via http://rsif.royalsocietypublishing.org. frog. Liu et al. [8] recently demonstrated levitation of a live mouse. In common with all ground-based techniques to simulate weightlessness, there are effects introduced by diamagnetic levitation that are not present in a weightless environment. For the first time, we critically assess the effect of diamagnetic levitation on a growing bacterial culture in liquid, over an $18 \mathrm{~h}$ period. We use a superconducting magnet to levitate the culture.

Guevorkian \& Valles [9] reported that Paramecia change their swimming behaviour in magnetically altered effective gravity, in response to the altered buoyancy of the cells. Coleman et al. [10] investigated the effect of magnetic levitation on growth and cell cycle changes in wild-type yeast cells, concluding that neither the growth nor the cell cycle was affected by the magnetic field when cells were levitated, but that growth was reduced at increased effective gravity. Some selective effects were seen on cells with specific mutation in transcription factors, known to mediate responses to environmental stresses such as gravity and shear stress, indicating that adaptive gene expression was required for the cells to be able to grow normally. Our previous experiments on magnetically levitated Arabidopsis thaliana cell cultures have also shown that adaptive responses occur, again detected by changes in the expression of 
transcription factors. In this case, the adaptations were similar to those seen when cells experienced simulated weightlessness in a random positioning machine [11]. (Wilson et al. [12] have also shown that space flight alters bacterial gene expression and virulence, in this case owing to a decrease in the levels of the global gene regulator Hfq, again indicating the need for adaptation to the conditions experienced during growth in a weightless environment.)

Here we show that magnetic levitation of bacteria in a liquid culture increases the rate of population growth and the final cell density of the culture. We investigate the mechanism leading to this enhancement.

\section{INITIAL HYPOTHESIS}

For these experiments, we chose Escherichia coli and Staphylococcus epidermidis as examples of human commensal bacteria, and as representatives of the Gramnegative and Gram-positive groups, respectively. We used a specially designed $17 \mathrm{~T}$ superconducting solenoid with a closed-circuit cryogenic system to levitate samples of bacterial culture in liquid nutrient broth. The magnet has a vertical bore. The temperature of the bore was kept at $37^{\circ} \mathrm{C}$ by forced air flow. Using a superconducting magnet to levitate biological organisms and material $[10,11,13-15]$ rather than a resistive magnet is attractive because we can levitate for periods much longer than can be obtained, economically, using a resistive magnet.

\subsection{Effective gravity acting on the liquid culture medium}

Water, being diamagnetic, is repelled from the strong magnetic field at the centre of the solenoid. The liquid levitates where the magnetic force balances the gravitational force, approximately $75-80 \mathrm{~mm}$ above the geometric centre of the solenoid, depending on the solenoid current [16,17]. Following Valles et al. [5], we define the effective gravity acting on the water as $\Gamma_{z}=\chi_{\mathrm{w}} B B_{z}^{\prime} /\left(\rho_{\mathrm{w}} \mu_{0}\right)-g$, where $B$ and $B_{z}^{\prime}=\partial B / \partial z$ are the magnitude of the magnetic field and the magnetic field gradient, respectively; $\chi_{\mathrm{w}}=-9 \times 10^{-6}$ (SI units) and $\rho_{\mathrm{w}}=1000 \mathrm{~kg} \mathrm{~m}^{-3}$ are the volume magnetic susceptibility and density of water, respectively; $g=9.8 \mathrm{~m} \mathrm{~s}^{-2}$ is the gravitational acceleration at the Earth's surface and $\mu_{0}=4 \pi \times 10^{-7} \mathrm{NA}^{-2}$. At the levitation point $\Gamma_{z}=0$. Since the culture medium is composed mostly of water, it levitates at the same position, under the same conditions. Note that a positive value of $\Gamma_{z}$ indicates a net upward force, and a negative value of $\Gamma_{z}$ indicates a net downward force. A more detailed discussion of effective gravity and the variation in $\Gamma$ near the levitation point can be found in the electronic supplementary material, appendix S1.

\subsection{Effective gravity acting on the cells}

Whether an individual cell floats or sinks in the liquid culture medium depends on Archimedes' principle, i.e. on the difference between the cell's weight and the weight of fluid displaced by the cell. In a weightless environment, e.g. in an orbiting spacecraft, both weights are zero, so the cells are neutrally buoyant. We express the net force acting on the cell, including buoyancy forces, as an effective gravity: $\Gamma_{z}^{(c)}=\Delta \chi B B_{z}^{\prime} /\left(\rho_{\mathrm{c}} \mu_{0}\right)-g \Delta \rho / \rho_{\mathrm{c}}$, where $\Delta \chi=\chi_{\mathrm{c}}-\chi_{\mathrm{w}}$ and $\Delta \rho=\rho_{\mathrm{c}}-\rho_{\mathrm{w}}$. Here, $\chi_{\mathrm{c}}$ is the spatially averaged volume magnetic susceptibility of the cell and $\rho_{\mathrm{c}}$ is the spatially averaged ('buoyant') density of the cell (i.e. its mass divided by its volume). See the electronic supplementary material, appendix S2.1, for the derivation and additional discussion.

For neutral buoyancy, we require the net force acting on the cell to be zero; that is, we require $\Gamma_{z}^{(c)}=0$. Outside the magnet, $\Gamma_{z}^{(c)} \approx-0.09 g$, since the buoyant density of the bacterial cells is $\rho_{\mathrm{c}} \approx 1090 \mathrm{~kg} \mathrm{~m}^{-3}$ [18]; hence, we expect the cells to sediment in the culture medium, outside the magnet. We now consider whether the diamagnetic force on the cells and on the fluid can prevent the cells from sinking. We estimate $\Delta \chi=-(8 \pm 3) \times 10^{-7}$ experimentally by measuring the levitation position of a bacterial pellet in the bore [19]; most of the experimental error in this measurement is owing to uncertainty in the water content of the bacterial pellet. From this result, we estimate that $\Gamma_{z}^{(c)}=(-0.01 \pm 0.03) g$ at the levitation point of the culture medium. This analysis suggests that it is possible to achieve a pseudo-weightless condition in the magnetically levitated bacterial culture, in the sense that the fluid medium is weightless and the cells are simultaneously neutrally buoyant in the fluid $\left(\Gamma_{z}^{(c)} \approx 0 g\right)$.

\subsection{Estimate of the effect of levitation on the sedimentation rate of the cells}

Based on our experimental measure of $\Delta \chi$, we estimate that the sedimentation rate should be reduced to $(10 \pm 30)$ per cent of the rate exhibited outside the magnet. The negative percentage encompassed by the uncertainty in this value indicates that our estimate includes the possibility that the cells will float to the surface, rather than sink. We have expressed the sedimentation rate as a percentage of the $1 g$ control rate, rather than an absolute value, since the rate is dependent on the cell size. The calculation is outlined in the electronic supplementary material, appendix S2.2.

\section{CONTROLS}

We use culture volumes of more than $1 \mathrm{ml}$ to allow for sampling during the experiment and to ensure that the culture volume is not significantly affected by evaporation. Strong magnetic fields of the order of $10 \mathrm{~T}$ are required to magnetically levitate culture volumes of this size; in our experiments, $B=12.3 \mathrm{~T}$ at the levitation point. There is evidence that $B$ fields of this strength affect biological organisms at the cellular level. For example, striking changes were observed in the orientation of cell-division cleavage planes in developing frog embryos in a static field $B \sim 1 \mathrm{~T}[20,21]$. Stresses can arise from a magnetic torque resulting from anisotropy in the magnetic susceptibility of structures $[21,22]$. The significance of these forces depends on whether the energy associated with such forces is 
larger than the thermal energy scale. Another possibility is that the magnetic field can affect biochemical kinetics [23]. Internal stresses in a biological cell can also be altered in a gradient magnetic field, owing to variations in the magnetic susceptibility of the cell's constituents. Valles et al. [5] performed experiments on magnetically levitated frog embryos, concluding that levitation reduced the gravity-induced internal stresses within the cell.

We shall not analyse these possibilities in further detail here. However, by using a control sample placed at the centre of the solenoid coil, enclosing the $\Gamma=1 \mathrm{~g}$ point, we can distinguish experimentally between the effects of magnetic forces that are proportional to the field-field gradient product $B B_{z}^{\prime}$, and magnetic effects that depend only on $B[5,24]$. As an additional control, a sample container was also placed below the centre of the solenoid, enclosing the $\Gamma=2 g$ point, where gravity and the magnetic force are additive, and $B=12.3 \mathrm{~T}$. For convenience, we label the sample containers ' $0 g^{*}$, ' $1 g^{*}$ ' and ' $2 g^{*}$ ' corresponding to the effective gravity enclosed by each container; the asterisk on the label indicates the sample is in a strong magnetic field, either $16.3 \mathrm{~T}$ at $1 g^{*}$ or $12.3 \mathrm{~T}$ at $0 g^{*}$ and $2 g^{*}$. The variation of $\Gamma$ in the $0 g^{*}$ container is discussed in the electronic supplementary material, appendix S1, and shown in the electronic supplementary material, figure $\mathrm{S} 1$. We use the label ' $1 g$ ' to indicate the control sample, grown outside the magnet.

\section{REDUCED SEDIMENTATION RATE}

To test our hypothesis that levitation inhibits sedimentation of the bacteria in the liquid culture, we used $E$. coli transformed to a green fluorescence protein (GFP) to visualize the distribution of cells within the culture vessel. Cultures were exposed to the magnetic field, one at each of the positions $0 g^{*}, 1 g^{*}$ and $2 g^{*}$, simultaneously at a temperature of $37^{\circ} \mathrm{C}$ for $18 \mathrm{~h}$. Figure 1 shows that the sedimentation rate of cells was reduced in the $0 g^{*}$ position, compared with the $1 g^{*}$ sample, indicated by the higher optical density of the $0 g^{*}$ culture throughout the tube. Sedimentation at the $2 g^{*}$ position was enhanced compared with the $1 g^{*}$ sample: the supernatant was almost clear, with the cells forming a layer on the bottom of the vessel. In separate experiments, the optical density $\left(\mathrm{OD}_{600 \mathrm{~nm}}\right) \mathrm{OD}_{1}$ of the supernatant was measured to determine the cell density remaining in suspension. A second optical density measurement $\mathrm{OD}_{2}$ was taken immediately after vortexing each sample to determine the OD value of an evenly suspended culture. The fraction of sedimented cells after the $18 \mathrm{~h}$ period is $S=1-\mathrm{OD}_{1} / \mathrm{OD}_{2}$, which is proportional to the sedimentation speed and the time of incubation. The $\mathrm{OD}_{1}$ of the $0 g^{*}$ culture was higher than that of the $1 g^{*}$ sample in the magnet, and the $1 g$ control outside the magnet. The fraction of sedimented cells at the $0 g^{*}$ position was $S\left(0 g^{*}\right)=0.38 \pm 0.06$, whereas in the $1 g^{*}$ control, $S\left(1 g^{*}\right)=0.54 \pm 0.01$. In the $1 g$ control outside the magnet, $S(1 g)=0.50 \pm$ 0.01 . The uncertainties are one standard deviation. These results indicate that significantly more bacteria

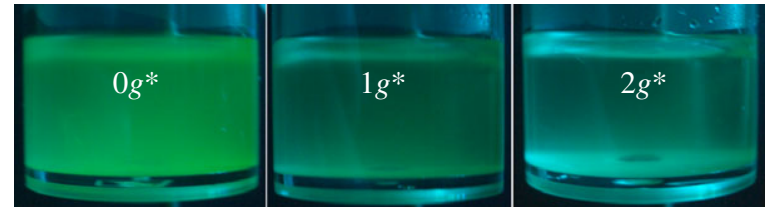

Figure 1. Sedimentation and growth of cultures in the magnet. Samples of E. coli (pSB2999) culture in $25 \mathrm{~mm}$ diameter containers, expressing the green fluorescence protein, were grown in the magnet at $0 g^{*}, 1 g^{*}$ and $2 g^{*}$ statically for $18 \mathrm{~h}$ at $37^{\circ} \mathrm{C}$ and then visualized under UV illumination. The liquid depth is $15 \mathrm{~mm}$.

remain in suspension in $0 g^{*}$ than in the $1 g$ and $1 g^{*}$ samples. There is a small difference between $S(1 g)$ and $S\left(1 g^{*}\right)$ which may be owing to some mixing as the $1 g^{*}$ sample is withdrawn from the magnetic field. The ratios $S\left(0 g^{*}\right) / S(1 g)$ and $S\left(0 g^{*}\right) / S\left(1 g^{*}\right)$ lie just outside the range we estimate from the Stokes drag analysis. This suggests that another mechanism may be influencing the apparent sedimentation rate. In $\S 7$, we discuss experiments in which we image the samples in situ. These experiments show that the gradient magnetic field can cause convective stirring where there is a gradient in the dissolved oxygen concentration.

\section{EFFECT ON GROWTH PHASES}

To investigate the effect of levitation on the growth of these cultures, cell density was measured as a function of time by taking optical density $\left(\mathrm{OD}_{600 \mathrm{~nm}}\right)$ measurements at approximately $1 \mathrm{~h}$ intervals to determine growth rate and lag time. Cultures of untransformed E. coli and S. epidermidis were grown within the magnet at the three different positions $\left(0 g^{*}, 1 g^{*}\right.$ and $\left.2 g^{*}\right)$. In addition, control samples were incubated outside the magnet $(1 g)$. Cultures within the magnet bore and the $1 g$ control sample were incubated statically at $37^{\circ} \mathrm{C}$. Each sample was mixed prior to measurement. Figure 2 shows that for both E. coli and S. epidermidis cultures grown at $0 g^{*}$, an overall enhancement of growth was apparent compared with the static $(1 \mathrm{~g})$ control cultures. No difference in the lag phase or initial growth rate was observed when cultures had low cell density. However, the cultures at $1 g^{*}$ and $2 g^{*}$ and the $1 g$ control all showed a lower initial growth rate than the $0 g^{*}$ cultures (figure 2). The final cell density in the $0 g^{*}$ samples was approximately 1.5 times that of the other cultures. These results are reproducible and statistically significant. The growth in the $1 g$ control is comparable with that in the $1 g^{*}$ sample, demonstrating that, in the absence of a magnetic field gradient, the magnetic field has no observable effect on the growth. For comparison, we performed an experiment on a shaken culture outside the magnet, in which we expect the liquid to be fully aerated. We found that, although growth was enhanced at $0 g^{*}$ compared with all the static cultures, it was not as high as that achieved by the aerated culture outside the magnet (table 1). Liquid loss via evaporation was insignificant because the culture vessels were airtight. 

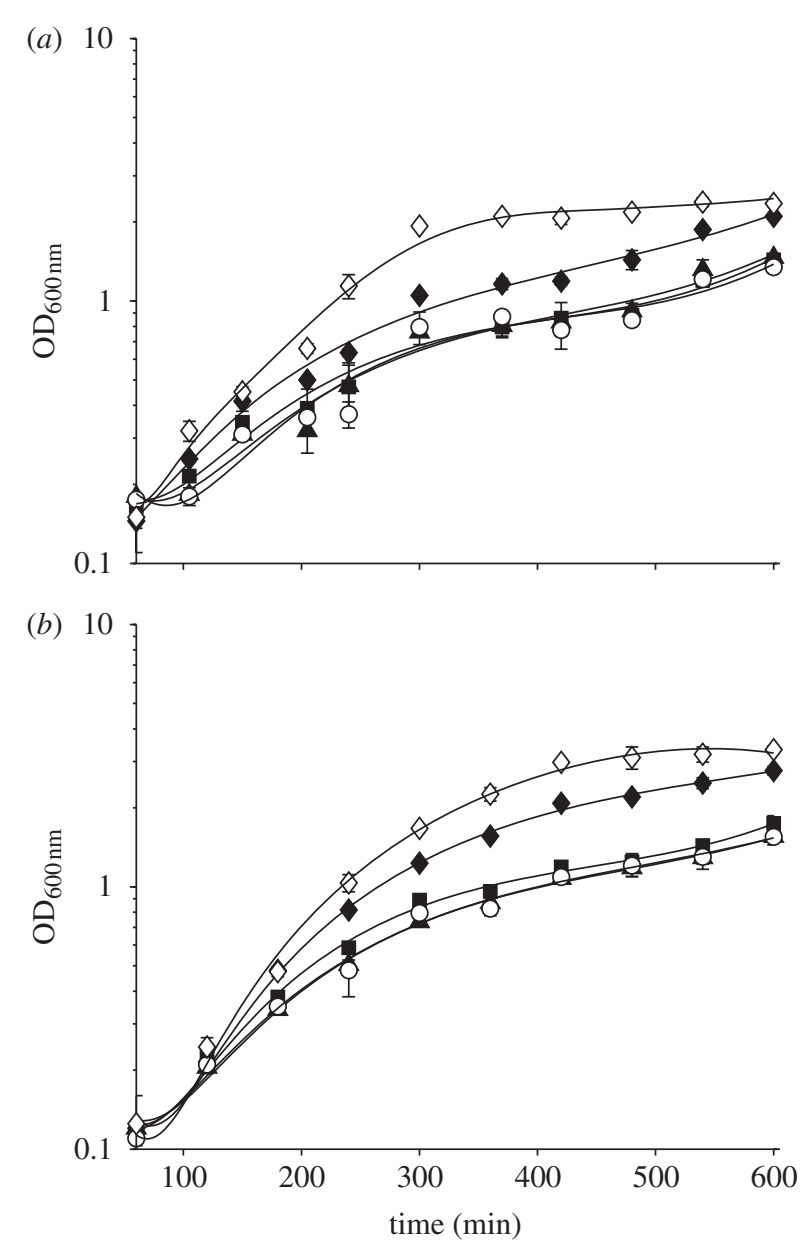

Figure 2. Growth of E. coli and S. epidermidis. Cultures of (a) E. coli and (b) S. epidermidis were grown at $37^{\circ} \mathrm{C}$ in nutrient broth. They were either exposed to altered effective gravity in the magnet bore (filled diamond, $0 g^{*}$; filled square, $1 g^{*}$; filled triangle, $2 g^{*}$ ) or were grown outside the magnet $(1 g)$ (open circle, statically; open diamond, aerated). Samples were collected at hourly intervals in order to determine the cell density, using $\mathrm{OD}_{600 \mathrm{~nm}}$. Error bars show 1 s.d.

\section{OXYGEN AVAILABILITY LIMITING GROWTH}

\subsection{Experiments with perfluorodecalin}

The lower growth rate in the static cultures, compared with the shaken cultures, suggests that availability of $\mathrm{O}_{2}$ is limiting growth in the static cultures. To test this experimentally, we performed experiments in tubes containing different volumes of liquid medium $V$. We also performed experiments in tubes of different diameters, to see the effect of varying the area of the air-liquid interface $A$. We found a clear positive relationship between final cell number density and $A$ / $V$ (figure 3). This result lends weight to the hypothesis that the availability of $\mathrm{O}_{2}$ is limiting growth in these experiments, since the $\mathrm{O}_{2}$ flux across the air-water interface is proportional to $A$ and the $\mathrm{O}_{2}$ concentration of the liquid resulting from this flux is inversely proportional to $V$. To confirm that availability of $\mathrm{O}_{2}$ is limiting growth, cultures were oxygenated from the bottom of the vessel by using a perfluorocarbon (PFC) artificial gas carrier: perfluorodecalin. PFC has a high saturation capacity for $\mathrm{O}_{2}$, and is both more dense than, and immiscible with, water; it forms a discrete layer at the bottom of the culture and has been shown previously to enhance growth of bacterial cultures [25]. All the samples with PFC showed enhanced growth compared with samples without PFC. Under these conditions, the final cell number density of the $0 g^{*}$ sample was as high as in the aerated cultures and the $1 g^{*}, 2 g^{*}$ and $1 g$ cultures all grew as well as the $0 g^{*}$ sample without PFC (table 1 ). This confirms that lack of $\mathrm{O}_{2}$ is limiting growth in the lower region of the static cultures. Further, these results suggest a reason for the $0 g^{*}$ growth enhancement: magnetic levitation increases the availability of $\mathrm{O}_{2}$. We test this hypothesis by a gene expression study in the following section. The fact that the growth rate of the $1 g^{*}$ culture is increased by inclusion of the PFC layer indicates that the magnetic field does not limit growth in these experiments.

\subsection{Changes in gene expression}

In adapting to different growth conditions, E. coli alters the composition of its respiratory pathways, changing the amount of different terminal oxidases to optimize its respiratory chain according to the substrates present and the physiological needs of the cell. Cytochrome $b_{\mathrm{o}}$, encoded by the cyoABCDE operon, operates at high oxygen concentration and has low affinity for oxygen. Expression of the cyo operon is decreased under anaerobic conditions by the global anaerobic regulators ArcA and Fnr [26]. The appB cytochrome bd-type oxidase has a high oxygen affinity and is encoded in an operon with appA ( $\mathrm{pH} 2.5$ acid phosphatase). Their expression is regulated by app $Y$ and stress-response sigma factor RpoS under microaerobic conditions, when $a p p B$ may be required for efficient electron transport [25]. We used microarray-based gene expression profiling to investigate the response to $\mathrm{O}_{2}$ depletion. RNA was extracted from each of the $E$. coli cultures grown under the different test conditions when the cells reached mid-exponential growth phase $\left(\mathrm{OD}_{600 \mathrm{~nm}}=0.5\right)$ and samples analysed on an E. coli oligonucleotide array (table 2). Expression of genes in the cyo operon were consistently approximately twofold upregulated in the $0 g^{*}$ samples compared with all other static samples. No significant difference in expression of these genes was detected between the $0 g^{*}$ static culture and the $1 g$ aerated culture. The opposite pattern was seen for genes in the app operon; the expression of these genes was enhanced at $2 g^{*}$. Additionally, the anaerobic growth regulator $f n r$ was downregulated in the $0 g^{*}$ compared with $2 g^{*}$ sample. In general, genes known to be associated with anaerobic adaptation were more highly expressed in samples with the lowest growth. Changes in expression of $c y o A$ and $a p p B$ were also confirmed by measuring mRNA levels using quantitative real-time reverse transcriptase polymerase chain reaction (qRT-PCR) analysis and showed that the expression of the cyoA gene was enhanced at $0 g^{*}$ compared with the control (table 3 ), with the 1.5-fold change observed being similar to results obtained from the microarray analysis. The 
Table 1. Effects of culture conditions on cell growth. Cultures were grown under different test conditions for $18 \mathrm{~h}$ at $37^{\circ} \mathrm{C}$ in the $0 g^{*}, 1 g^{*}$ and $2 g^{*}$ positions. The $1 g$ control samples were grown outside the magnet either statically $(1 g)$ or with shaking ( $1 \mathrm{~g}$ aerated). Optical density $\left(\mathrm{OD}_{600 \mathrm{~nm}}\right)$ of samples was determined after $18 \mathrm{~h}$. (a) Final optical densities of cultures of $E$. coli grown without and with $\mathrm{O}_{2}$-gassed perfluorodecalin (PFC) layer at the bottom of the culture. (b) Ratios of final cell density values of $E$. coli and $S$. epidermidis cultures. Only E. coli static cultures were oxygenated from the bottom of the culture by adding $\mathrm{O}_{2}$-gassed PFC. n.d. not determined.

\begin{tabular}{|c|c|c|c|}
\hline \multirow[b]{2}{*}{ condition } & \multicolumn{3}{|c|}{ final cell density $\left(\mathrm{OD}_{600 \mathrm{~nm}}\right)$} \\
\hline & without PFC & with PFC & \\
\hline \multicolumn{4}{|l|}{$(a)$} \\
\hline $0 g^{*}$ & $1.9 \pm 0.3$ & $2.3 \pm 0.1$ & \\
\hline $1 g^{*}$ & $1.4 \pm 0.1$ & $1.9 \pm 0.1$ & \\
\hline $2 g^{*}$ & $1.3 \pm 0.2$ & $1.8 \pm 0.1$ & \\
\hline $1 g$ & $1.3 \pm 0.1$ & $1.8 \pm 0.1$ & \\
\hline $1 g$ aerated & $2.4 \pm 0.2$ & n.d. & \\
\hline \multicolumn{4}{|l|}{ (b) } \\
\hline test comparison & E. coli & E. coli with PFC & S. epidermidis \\
\hline $0 g^{*} / 1 g$ & $1.73 \pm 0.35$ & $1.24 \pm 0.05$ & $1.94 \pm 0.16$ \\
\hline $1 g^{*} / 1 g$ & $1.09 \pm 0.03$ & $1.06 \pm 0.07$ & $1.17 \pm 0.25$ \\
\hline $2 g^{*} / 1 g$ & $1.04 \pm 0.08$ & $1.01 \pm 0.02$ & $0.73 \pm 0.11$ \\
\hline $0 g^{*} / 1 g^{*}$ & $1.59 \pm 0.29$ & $1.16 \pm 0.03$ & $1.53 \pm 0.16$ \\
\hline $0 g^{*} / 1 g$ aerated & $0.9 \pm 0.05$ & n.d. & $0.81 \pm 0.02$ \\
\hline
\end{tabular}
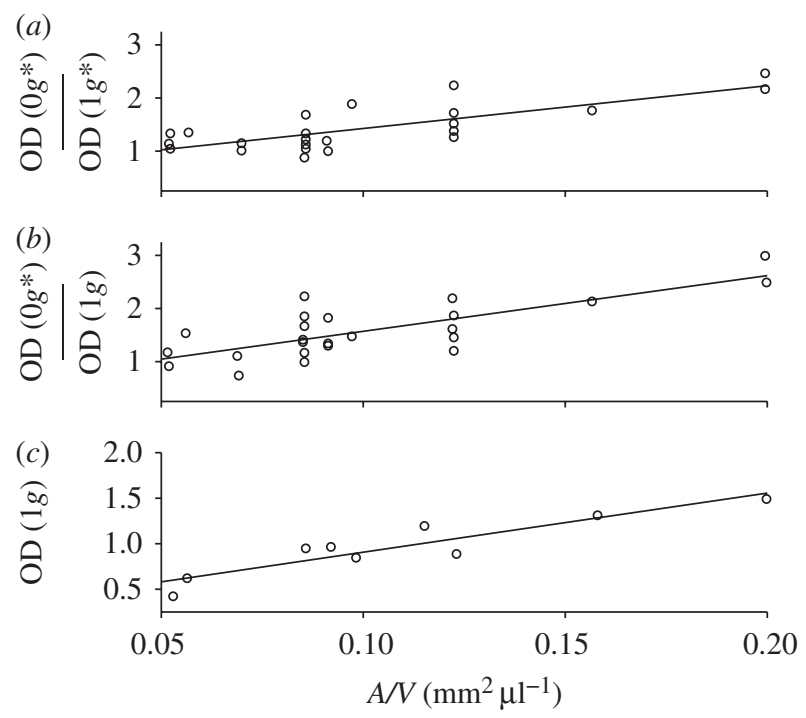

Figure 3. Relationship between final cell density and liquidair interface area/sample volume. Samples were incubated for $18 \mathrm{~h}$ in tubes with different dimensions (see the electronic supplementary material, table S1). The optical density $\left(\mathrm{OD}_{600 \mathrm{~nm}}\right)$ after $18 \mathrm{~h}$ shows a clear increase with $A / V ; A$ is the area of the liquid-air interface and $V$ is the sample volume. (a) OD of the $0 g^{*}$ sample, $\mathrm{OD}\left(0 g^{*}\right)$, relative to the OD of the $1 g^{*}$ control sample in the magnet, $\mathrm{OD}\left(1 g^{*}\right)$. (b) OD of the $0 g^{*}$ sample, $\mathrm{OD}\left(0 g^{*}\right)$, relative to the OD of the $1 g$ control outside the magnet, $\mathrm{OD}(1 g)$. (c) OD of the $1 g$ control. The scatter in the data points gives a good indication of the experimental uncertainty. Lines show the least-squares linear fit to the data. The gradients of the lines are, including standard errors, $8 \pm 2,11 \pm 2$ and $6.5 \pm 1.0 \mu \mathrm{l} \mathrm{mm}{ }^{-2}$ for the data in $(a),(b)$ and $(c)$, respectively.

expression of the $a p p B$ gene was downregulated at $0 g^{*}$ but was enhanced at the $2 g^{*}$ position compared with the control by approximately twofold. Importantly, no differences in level of expression of these cytochromes were found between the $1 g^{*}$ and $1 g$ static samples, indicating that their expression was not influenced by the magnetic field or by differences in distribution of other nutrients influenced by the magnetic field, but rather by differences in oxygen availability under the different test conditions.

\section{MAGNETICALLY INDUCED CONVECTION}

One might speculate that the increase in oxygen availability in the levitated $\left(0 g^{*}\right)$ sample may be owing to the observed reduction in the rate of sedimentation. This allows bacteria to remain closer to the surface where the liquid is enriched by oxygen diffusing across the air-liquid interface. While this is a plausible hypothesis, we also investigated whether previous studies of magnetically induced convection in water could be relevant [13,27-32]. Molecular oxygen is paramagnetic and is attracted to a magnetic field: molecular oxygen above the solenoid is pulled towards the centre of the solenoid, where the field is largest. This force enhances the buoyancy of objects immersed in a paramagnetic fluid [33-36]. Although the paramagnetism of $\mathrm{O}_{2}$ at room temperature is weaker than that of liquid oxygen, the paramagnetic force on the $\mathrm{O}_{2}$ molecules in air in the bore of our superconducting magnet is nevertheless significant owing to the large gradient magnetic field. This is easily demonstrated by levitating a droplet of water in the magnet: in air, the droplet levitates $6 \mathrm{~mm}$ higher than its position in nitrogen gas (at the same temperature and pressure) owing to the additional buoyancy force provided by the paramagnetic force on the $\mathrm{O}_{2}$ molecules in the air [17]. Ueno [37] showed that the paramagnetic force on $\mathrm{O}_{2}$ in the air is strong enough to extinguish a burning candle with a $1.5 \mathrm{~T}$ 
Table 2. Microarray analysis of changes in expression of cytochromes and fnr. Comparison of significant fold changes in expression for genes involved in cytochrome regulation in E. coli K12 MG1655. Samples were exposed to altered effective gravity at $37^{\circ} \mathrm{C}$ in the magnet bore $\left(0 g^{*}, 1 g^{*}\right.$ or $\left.2 g^{*}\right)$ or grown outside the magnet $(1 g)$ either statically or aerated. All samples were harvested at $\mathrm{OD}_{600 \mathrm{~nm}}=0.5$ for RNA extraction to minimize effects caused by changes in cell population and associated depletion of gases and nutrients. Total RNA was extracted from four independent replicates and used for microarray analysis. Data were analysed using GeneSpring GX 7.3, and normalized against 50\% of signal for each independent array and filtered using $p$-value $=0.05$.

\begin{tabular}{llll}
\hline test conditions & gene & gene product & fold change \\
\hline $0 g^{*}$ versus $1 g^{*}$ & cyoA & cytochrome o ubiquinol oxidase subunit II & 2.11 \\
& cyoB & cytochrome o ubiquinol oxidase subunit I & 2.34 \\
& appA & phosphoanhydride phosphorylase & 0.47 \\
$0 g^{*}$ versus $2 g^{*}$ & $a p p C$ & cytochrome bd-II oxidase, subunit I & 0.45 \\
& cyoB & cytochrome o ubiquinol oxidase subunit I & 2.98 \\
& $a p p B$ & cytochrome bd-II oxidase, subunit II & 0.33 \\
$0 g^{*}$ versus $1 g$ & appC & cytochrome bd-II oxidase, subunit I & 0.2 \\
& Fnr & DNA-binding transcriptional dual regulator, global regulator of anaerobic growth & 0.43 \\
& $c y o D$ & cytochrome o ubiquinol oxidase subunit IV & 2.12 \\
$1 g^{*}$ versus $1 g$ aerated & protoheme IX farnesyltransferase & 2.12 \\
$1 g^{*}$ versus $2 g^{*}$ & Fnr $B$ & DNA-binding transcriptional dual regulator, global regulator of anaerobic growth & 0.37 \\
& $a p p B$ & cytochrome o ubiquinol oxidase subunit I & 0.42 \\
\hline
\end{tabular}

Table 3. Comparison of qRT-PCR and microarray data for $c y o A$ and $a p p B$. Samples of RNA extracted for the array experiments from four independent replicates of cells grown in altered effective gravity at $37^{\circ} \mathrm{C}$ in the magnet bore $\left(0 g^{*}\right.$, $1 g^{*}$ or $\left.2 g^{*}\right)$ or statically outside the magnet $(1 g)$ were used for qRT-PCR analysis. Cells were recovered from each culture for RNA extraction when $\mathrm{OD}_{600 \mathrm{~nm}}=0.5$. Levels of $c y o A$ and $a p p B$ were compared in each case with the $1 g$ control sample. Details of TaqMan probes and dyes used are provided in the electronic supplementary material, table S3. Values that were statistically different at the $5 \%$ level from a $1: 1$ ratio are indicated by an asterisk. ${ }^{*} p$-values $<0.05$.

\begin{tabular}{|c|c|c|c|c|}
\hline \multirow[b]{3}{*}{ test conditions } & \multicolumn{4}{|c|}{ fold change } \\
\hline & \multicolumn{2}{|l|}{ cyoA } & \multicolumn{2}{|l|}{$a p p B$} \\
\hline & arrays & qRT-PCR & arrays & qRT-PCR \\
\hline $0 g^{*} / 1 g$ & $1.49^{*}$ & $1.57^{*}$ & 0.69 & 0.80 \\
\hline $1 g^{*} / 1 g$ & 0.71 & 0.90 & 0.88 & 0.92 \\
\hline $2 g^{*} / 1 g$ & 0.95 & 0.80 & $1.82^{*}$ & $2.20^{*}$ \\
\hline
\end{tabular}

electromagnet. $\mathrm{O}_{2}$ dissolved in the liquid culture medium is similarly attracted by the magnetic field [38]. Since $\mathrm{O}_{2}$ in the liquid is consumed by the bacteria and replaced at the liquid-air interface (from the $\mathrm{O}_{2}$ in the air above the liquid), an $\mathrm{O}_{2}$ concentration gradient results, producing a corresponding gradient in the magnetic force density. Analogous magnetic effects on convection have been observed in crystal growth experiments in high gradient magnetic fields, resulting from a concentration gradient of solute $[39,40]$.

\subsection{Theory}

We now consider whether this force gradient can cause convection of the liquid medium by comparison with convection driven by a temperature-dependent density gradient, which is well understood. We define an effective liquid density $P$ such that the net vertical force on a volume $V$ of liquid in the magnetic field $F_{z}=\chi V B B_{z}^{\prime} / \mu_{0}-\rho_{\mathrm{w}} V g$ can be written $F_{z}=P V \Gamma$, where $P=\rho_{\mathrm{w}}+\left(\chi-\chi_{\mathrm{w}}\right) B B_{z}^{\prime} /\left(\Gamma \mu_{0}\right) ; \rho_{\mathrm{w}}$ and $\chi$ are the liquid density (approximately the same as water) and the volume magnetic susceptibility, respectively. For simplicity, we consider the forces on-axis only, where $\Gamma=\Gamma_{z}$. Importantly, note that $\chi$ depends on the concentration $C$ (here, measured in molecules per $\mathrm{m}^{3}$ ) of dissolved oxygen in the liquid: $\chi(C)=\chi_{\mathrm{w}}+\gamma C$, where $\gamma=43 \times 10^{-9} \mathrm{~m}^{3} \mathrm{~mol}^{-1} / N_{A}$ is the molecular magnetic susceptibility of $\mathrm{O}_{2}$ at room temperature [41]; $N_{A}$ is Avagadro's number. Therefore, the effective density of the liquid $P$ depends on the concentration of dissolved oxygen $C$. A gradient in the oxygen-dependent effective density can give rise to convection in an entirely analogous way to a temperature-dependent density gradient. In the text-book problem of Rayleigh-Bénard (RB) convection (e.g. [42]), a temperature gradient is established between a hot plate at the base of the liquid and a cold plate at the top. The difference in density between the warm liquid near the base and the cooler liquid at the top causes the system to become unstable to convection when the temperature gradient exceeds a critical value, determined by the Rayleigh number. For example, for $20^{\circ} \mathrm{C}$ water between plates separated by $1 \mathrm{~cm}$, we expect to observe convection when the temperature gradient exceeds approximately $0.15 \mathrm{~K} \mathrm{~cm}^{-1}$. We now consider an analogous configuration, which will allow us to use the existing theory on RB convection to provide an insight into the present problem: we assume for the moment that the bacteria lie in a layer at the bottom of the vessel, consuming $\mathrm{O}_{2}$, so that, in equilibrium, $C$ decreases linearly from $C_{\mathrm{i}}$ at the liquid-air interface to $C_{\mathrm{b}}$ at the container bottom. We can define a Rayleigh number for this configuration, in an exact analogy with the thermal 
convection problem,

$$
R a^{*}=\frac{\alpha \Gamma h^{3} \Delta C}{D v} .
$$

Here, $\Delta C=C_{\mathrm{b}}-C_{\mathrm{i}}, h \sim 1 \mathrm{~cm}$ is the depth of the liquid, $D \sim 3 \times 10^{-9} \mathrm{~m}^{2} \mathrm{~s}^{-1}$ is the diffusivity of $\mathrm{O}_{2}$ molecules in the medium at $37^{\circ} \mathrm{C}$ (approximately the same as that in water; [43]), $v \sim 1 \times 10^{-6} \mathrm{~m}^{2} \mathrm{~s}^{-1}$ is the kinematic viscosity of the medium and $\alpha$ is the fractional change of $P$ with $C$,

$$
\alpha=-\frac{1}{P}\left(\frac{\partial P}{\partial C}\right)
$$

Note that the molecular diffusivity $D$ and the concentration difference $\Delta C$ are the analogues of the thermal diffusivity and temperature difference, respectively, in the thermal convection problem. Poodt et al. [44] used a Rayleigh number for analysing the results of the similar problem of mass-transport in magnetically levitated crystal growth, although they do not introduce the concept of effective density explicitly. We emphasize that $R a^{*}$ is not the same as the 'magnetic Rayleigh number' that characterizes convection driven by temperature-induced changes in fluid magnetization (e.g. [45]). In the present problem, the $\mathrm{O}_{2}$ concentration gradient is responsible for convection, not the temperature, which is uniform throughout the liquid. Since the diffusivity of $\mathrm{O}_{2}$ in air is $\sim 1 \times 10^{4}$ times larger than in water [46], we can assume that there is a negligible $\mathrm{O}_{2}$ concentration gradient in the air above the liquid. Hence, we assume that the concentration of $\mathrm{O}_{2}$ in the surface liquid is approximately the same as that in water in equilibrium with the air, which is $C_{0} \sim 1 \times 10^{23}$ molecules per $\mathrm{m}^{3}$ at $37^{\circ} \mathrm{C}$ [47]. At the container bottom, the bacteria consume $\mathrm{O}_{2}$ until its concentration reaches $C_{\mathrm{b}} \approx 0.01 C_{0}$, at which point the low $\mathrm{O}_{2}$ concentration limits the activity of the bacteria ([48]; at these cell densities, $5 \times 10^{7}-5 \times 10^{9} \mathrm{~m}^{-3}$, the rate of $\mathrm{O}_{2}$ diffusion through the liquid limits the bacterial $\mathrm{O}_{2}$ consumption). For simplicity, we use the approximation $C_{\mathrm{b}}=0$. In the $0 g^{*}$ position, the effective density $\mathrm{P}$ of the $\mathrm{O}_{2}$-rich liquid at the surface is greater than that of the $\mathrm{O}_{2}$-poor region below. From existing theory on RB convection, we know that this configuration is unstable above $R a^{*} \sim 2000$. (Note that the culture at $2 g^{*}$ is stable against this type of convection since the paramagnetic force on the $\mathrm{O}_{2}$ pulls upward in this location.) Calculating the $R a^{*}$ number for the model system, we find that, within the $0 g^{*}$ sample, $R a^{*}>1 \times 10^{6}$ on-axis, assuming a constant 'mean' $\Gamma$ in the range $0.01 \mathrm{~g}$ to $0.1 \mathrm{~g}$, in this simple model. Hence, we expect our model $0 g^{*}$ system to be unstable to convection. Although, in the experiments, a fraction of the bacteria is suspended in the liquid, as we demonstrated in $\S 4$, and the $E$. coli are also motile, the large $R a^{*}$ number for the model $0 g^{*}$ system suggests that convection driven by an $\mathrm{O}_{2}$ concentration gradient could be possible under experimental conditions, too.

\subsection{Experiment to test for magnetically induced convection}

We tested this hypothesis experimentally by performing growth experiments on E. coli including the redox dye resazurin. This blue, water-soluble, dye is reduced, irreversibly, to the pink dye resorufin by reactions associated with bacterial respiration [49]. Resorufin can itself be further reduced to the colourless hydroresorufin by the same processes. However, the second reaction is reversible by $\mathrm{O}_{2}$ [49]. Since the dye molecules are diamagnetic, the magnetic force on each is small compared with the force on an $\mathrm{O}_{2}$ molecule; we measured the susceptibility of the powdered dyes using the Gouy method. Therefore, we do not expect the introduction of the dye to have any effect on the convection. Figure 4 shows cultures containing the dye in the $0 g^{*}$ and $2 g^{*}$ positions, and in a control sample $(1 g)$ outside the magnet. The photographs were obtained in situ, $6 \mathrm{~h}$ after introduction of the dye. Unlike the experiment performed to measure growth, where samples were mixed prior to sampling, these static cultures were left undisturbed. Just after the dye was introduced, all samples had a uniform blue colour, but turned pink after approximately $2 \mathrm{~h}$ owing to bacterial aerobic respiration. The pink colour of the $1 g$ control sample faded to colourless after a further $1-2 \mathrm{~h}$, as the dissolved $\mathrm{O}_{2}$ was depleted and the resorufin was further reduced. However, close to the air-liquid interface, the liquid remained pink owing to $\mathrm{O}_{2}$ diffusion across the interface, which prevented the reduction of resorufin to hydroresorufin. In contrast, the colour of the sample in the $0 g^{*}$ position did not fade to colourless, remaining bright pink (including in the region at the bottom of the container), except for the appearance of two paler oval-shaped areas. Comparison with the control sample suggests that within these pale ovals, the $\mathrm{O}_{2}$ concentration must be lower than in the rest of the fluid. We also note that the oval shapes of these regions are reminiscent of the shape of stagnant regions in thermal convection cells [42]. These two observations suggest that the concentration of dissolved $\mathrm{O}_{2}$ outside the oval regions is increased by convective transport of $\mathrm{O}_{2}$ from the relatively $\mathrm{O}_{2}$-rich surface liquid to the rest of the sample. The fluid within the ovals stagnates, as is typical in a convection cell, and therefore $\mathrm{O}_{2}$ levels in these regions are depleted by bacterial respiration, allowing resorufin to be reduced to its colourless form. Since the paramagnetic force on the $\mathrm{O}_{2}$ in the $2 g^{*}$ sample acts upwards, we expect these samples to be stable against paramagnetic $\mathrm{O}_{2}$ convection. This is evident from the well-defined pink layer at the top of this container.

Since the nutrient broth contains paramagnetic metal ions, $\mathrm{Fe}^{2+}, \mathrm{Cu}^{2+}$ and $\mathrm{Mn}^{2+}$, in concentrations comparable with $C_{0}$, one might ask whether these ions could also give rise to convection, since the bacteria require these ions for their metabolic processes. However, Bovallius \& Zacharias [50] showed that, in nutrient broth, these ions are present at sufficient concentration to avoid rate-limiting the bacterial growth. Hence, we do not expect a significant concentration gradient of these ions to arise; we emphasize that a concentration gradient is required to generate convection by this mechanism. Conversely, since we have shown that $\mathrm{O}_{2}$ depletion limits growth in our 

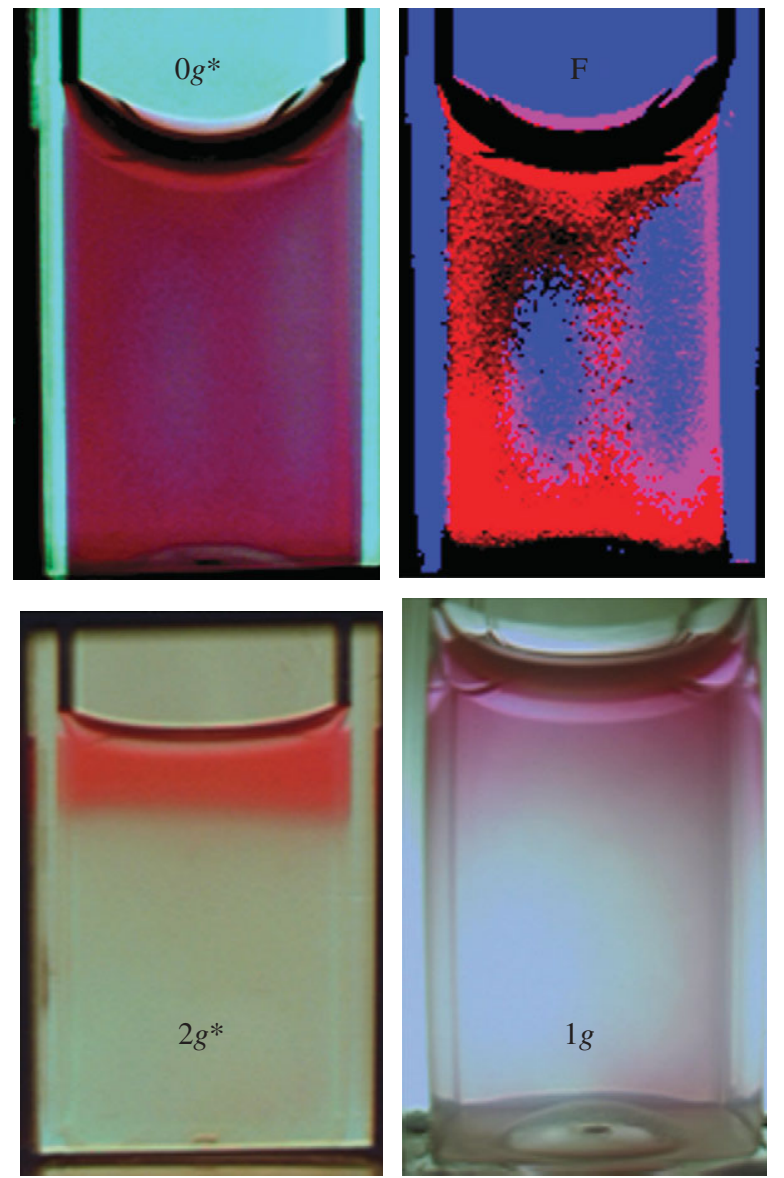

Figure 4. Magnetic convection in a bacterial culture. Static bacterial cultures $(E$. coli) in $10 \mathrm{~mm} \times 10 \mathrm{~mm} \times 45 \mathrm{~mm}$ cuvettes, containing resazurin dye. The liquid depth is $12 \mathrm{~mm}$. Cuvettes in the $0 g^{*}$ and $2 g^{*}$ positions in the magnet, and $1 g$ control outside the magnet, photographed in situ after $6 \mathrm{~h}$. The dye turns pink in regions where bacteria are undergoing aerobic metabolism but have not become anaerobic, highlighting areas with a relatively high $\mathrm{O}_{2}$ concentration. The image top right, labelled ' $\mathrm{F}$ ', is a false-colour version of the $0 g^{*}$ image shown top left. In this image, the $\mathrm{O}_{2}$-depleted regions are coloured blue. The shapes of these regions are reminiscent of the shape of thermal convection cells.

experiments, we expect a significant $\mathrm{O}_{2}$ concentration gradient. We have shown, theoretically and experimentally, how such an $\mathrm{O}_{2}$ concentration gradient in the magnetic field can generate convection.

A natural question to ask at this point is whether the sedimentation itself is inhibited by the paramagnetic convective stirring, rather than by diamagnetic levitation. Since we observe an increase in the rate of sedimentation in the $2 g^{*}$ position, this demonstrates that the diamagnetic force on the liquid and the cells does indeed have a direct effect on sedimentation (increasing the sedimentation rate in the $2 g^{*}$ sample), since the liquid in this position is stable against the paramagnetically induced convection discussed above. However, we must conclude that the convective stirring has a significant effect on the availability of $\mathrm{O}_{2}$ to the bacteria in the $0 g^{*}$ sample, since the convective flow transports $\mathrm{O}_{2}$ from the $\mathrm{O}_{2}$-rich surface through the bulk of the liquid.

\section{CONCLUSION}

From our experimental data, we attribute the increase in bacterial growth rate and higher final cell density in the $0 g^{*}$ samples to greater oxygen availability. The effect becomes enhanced at high cell density when the respiration of the bacteria rapidly depletes oxygen levels. This explanation is supported by the observations that (i) the $0 g^{*}$ sample supplemented with PFC behaves as a uniformly aerated culture and (ii) the anaerobic adaptation genes were more strongly induced in the $1 g^{*}$ and $2 g^{*}$ cultures, confirming that they were experiencing oxygen depletion. We have shown that the diamagnetic force can directly affect the sedimentation rate of a bacterial culture. We have also demonstrated that the gradient magnetic field in the $0 g^{*}$ position has a significant effect on the transport of $\mathrm{O}_{2}$ in the liquid culture: the consumption of $\mathrm{O}_{2}$ by the living cells and its replenishment by $\mathrm{O}_{2}$ diffusing across the liquid-air interface can generate convection in the magnetic field, analogous to the thermal, RB convection process. It is probable that the enhanced availability of $\mathrm{O}_{2}$ in $0 g^{*}$ is owing to this latter effect. Diamagnetic levitation has the potential to be a powerful technique to study the effects of weightlessness on biological cells, to complement existing Earth-based techniques such as clinorotation and random positioning. However, for diamagnetic levitation to be a useful model of the weightless space environment, where density-driven convective transport is absent, paramagnetically driven convection of $\mathrm{O}_{2}$ should be prevented. One possibility is to perform experiments on anerobically metabolizing organisms, or in non-liquid cultures [51].

\section{MATERIAL AND METHODS}

\subsection{Bacterial cultures}

Escherichia coli K12 MG1655 and S. epidermidis NCTC11047 were grown in nutrient broth (NB; Oxoid, UK). $\mathrm{Gfp}^{+}$bacteria were $E$. coli

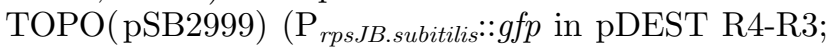
P.J. Hill, University of Nottingham) which were grown in NB supplemented with ampicillin (50 $\left.\mu \mathrm{gml}^{-1}\right)$. All cultures were grown at $37^{\circ} \mathrm{C}$. Aerated cultures were shaken at 150 r.p.m.

\subsection{Experimental levitation magnet system}

The superconducting solenoid has a $50 \mathrm{~mm}$ diameter vertical bore, open to the laboratory at both ends (see the electronic supplementary material, figure S2). A closed-cycle coolant system allows the magnet to run at high magnetic field on time scales of the order of several months. A constant mean temperature of $37^{\circ} \mathrm{C}$ in the bore was maintained, with variation over time less than $0.2^{\circ} \mathrm{C}$, by temperature-regulated forced air flow with feedback control. The temperature-controlled chamber consists of an acrylic tube (length $60 \mathrm{~cm}$, internal diameter $44 \mathrm{~mm}$ ), inserted into the magnet bore, containing three specimen tubes $(25 \mathrm{~mm}$ internal diameter, $25 \mathrm{ml}$ capacity), one at each of the $0 g^{*}, 1 g^{*}$ and $2 g^{*}$ positions (see the electronic supplementary 
material, figure $\mathrm{S} 3$ ). The $1 g^{*}$ sample is located at the centre of the coil; the $0 g^{*}$ and $2 g^{*}$ samples are located $75 \mathrm{~mm}$ above and below the $1 g^{*}$ position, respectively. The temperature of each sample was monitored by a thermocouple in contact with each specimen tube. The effect of magnetic field up to $17 \mathrm{~T}$ on the thermocouples was negligible.

\subsection{Growth experiments}

Cultures were inoculated into fresh $\mathrm{NB}$ at an $\mathrm{OD}_{600 \mathrm{~nm}}=0.05$. The growth experiments and sedimentation experiments presented in $\S \S 4$ and 5 were performed in $25 \mathrm{ml}$ containers $(25 \mathrm{~mm}$ internal diameter, $50 \mathrm{~mm}$ tall). The containers were filled with the liquid culture to a depth of $15 \mathrm{~mm}$. The experiments to determine the effect of the liquid surface area to volume ratio ( $(6)$ were performed in containers of varying sizes and culture volumes; their dimensions are summarized in the electronic supplementary material, table S1. Samples were exposed to $0 g^{*}, 1 g^{*}$ or $2 g^{*}$ for varying periods of time, in the dark at $37 \pm 0.1^{\circ} \mathrm{C}$. Each sample was removed from the magnet, mixed, and its cell numbers determined at approximately $1 \mathrm{~h}$ intervals to determine growth rate and lag time. Cell number was determined by viable count or by optical density $\left(\mathrm{OD}_{600 \mathrm{~nm}}\right)$. In the experiments to visualize convection $(\S 7)$, spectrophotometer cuvettes $(10 \mathrm{~mm} \times$ $10 \mathrm{~mm} \times 45 \mathrm{~mm}$ ) were filled with the liquid culture to a depth of $12 \mathrm{~mm}$ and incubated at $0 g^{*}, 1 g^{*}, 2 g^{*}$ and $1 \mathrm{~g}$ at $24^{\circ} \mathrm{C}$; the lower temperature slowed bacterial growth to allow changes within the fluid to be visualized more easily. Resazurin was used at a final concentration of $67 \mathrm{mg} \mathrm{ml}^{-1}$.

\subsection{Perfluorocarbon}

Perfluorodecalin (Flutec PP6) liquid was oxygenated for 20 min with 100 per cent oxygen gas and dispensed as $4 \mathrm{ml}$ aliquots in sample tubes. For growth experiments, $4 \mathrm{ml}$ of bacterial suspension was overlaid on the PFC and samples exposed to altered gravity in the magnet for $16 \mathrm{~h}$. The position of the sample in the magnet bore was altered to compensate for the raised height of the growth medium owing to the presence of the PFC layer in the bottom of the tube. Data were analysed using the one-way ANOVA and Tukey's pair-wise comparisons post hoc analysis with a $95 \%$ CI, using SPSS software.

\subsection{Microarray analysis}

Samples were exposed to each test condition and cells harvested in the mid-exponential phase of growth and four independent replicates of each experiment were performed. Total RNA were extracted and labelled using the protocol outlined in MessageAmp II-Bacteria. RNA (700 ng) was labelled with 5-(3-aminoallyl)-UTP. Samples $(5 \mu \mathrm{g})$ of RNA were labelled with Cy5 using NHS-ester reactive dye packs and purified using RNeasy MinElute columns. The DNA reference samples $(1 \mu \mathrm{g})$ were labelled using the Invitrogen 'BioPrime DNA labelling system', with Cy3-dCTP. Agilent MG1655 microarray slides were hybridized at $65^{\circ} \mathrm{C}$ for
$17 \mathrm{~h}$ and scanned at $5 \mu \mathrm{m}$ resolution using the extended dynamic range (high 100\%, low 10\%). Data were analysed using Agilent Feature Extraction software and imported into the GENESPRING GX package, normalized per chip to 50 per cent of signal and genes whose normalized expression levels changed twofold or more were identified by applying a $t$-test $(p=0.05)$. Differentially transcribed genes were divided into functional groups using COG and KEGG. A complete analysis of genes involved in oxidative phosphorylation pathway with a significantly different level of expression is presented in the electronic supplementary material, table S2.

\subsection{Quantitative real-time polymerase chain reaction ( $q R T-P C R)$}

All qRT-PCR experiments were performed in triplicate on RNA used for microarray analysis and cDNA was synthesized from $2 \mu \mathrm{g}$ RNA. Custom TaqMan assays for $c y o A$, appB and $e n v Z$ genes were used (see the electronic supplementary material, table S3). The thermal profile employed was $50^{\circ} \mathrm{C}$ for $2 \mathrm{~min}, 95^{\circ} \mathrm{C}$ for $10 \mathrm{~min}$, and 40 cycles of $95^{\circ} \mathrm{C}$ for $15 \mathrm{~s}, 60^{\circ} \mathrm{C}$ for $1 \mathrm{~min}$. Average $C_{\mathrm{T}}$ values and standard deviation were calculated for the three replicates. $\Delta \Delta C_{\mathrm{T}}$ was calculated by the difference between $\Delta C_{\mathrm{T}}$ of test samples $\left(0 g^{*}, 1 g^{*}, 2 g^{*}\right.$ and $1 g$ aerated) and control sample ( $1 g$ static).

C.E.D., O.L., R.J.A.H. and P.A. performed experiments; C.E.D., O.L., R.J.A.H., P.A., M.R.D., L.E. and C.E.D.R. designed experiments; C.E.D., O.L., C.E.D.R. and R.J.A.H. analysed data; and C.E.D., O.L., R.J.A.H., L.E. and C.E.D.R. wrote the paper. We thank the technical staff in the School of Physics, especially D. A. Holt, P. Smith, S. R. Booth, S. Nankervis and I. Taylor, for assistance in constructing the temperature-controlled chamber and in situ imaging hardware, and P. J. Hill for supplying the GFP bacteria. This work was supported by a Basic Technology Grant from EPSRC, UK; grant nos. GR/S83 005/01 and $\mathrm{EP} / \mathrm{G} 037647 / 1$.

\section{REFERENCES}

1 Cogoli, A. 2006 Cell biology. In Fundamentals of space biology: research on cells, animals, and plants in space (eds G. Clément \& K. Slenzka). Space Technology Library, vol. 18, pp. 121-170. El Segundo, CA: Microcosm Press/Springer.

2 van Loon, J. J. W. A. 2007 The gravity environment in space experiments. In Biology in space and life on earth: effects of spaceflight on biological systems (ed. E. Brinckmann), pp. 17-32. Weinheim, Germany: Wiley-VCH.

3 Beaugnon, E. \& Tournier, R. 1991 Levitation of water and organic substances in high static magnetic-fields. J. Phys. III 1, 1423-1428. (doi:10.1051/jp3:1991199)

4 Berry, M. V. \& Geim, A. K. 1997 Of flying frogs and levitrons. Eur. J. Phys. 18, 307-313. (doi:10.1088/0143-0807/ $18 / 4 / 012)$

5 Valles Jr, J. M., Lin, K., Denegre, J. M. \& Mowry, K. L. 1997 Stable magnetic field gradient levitation of Xenopus laevis: toward low-gravity simulation. Biophys. J. 73, 1130-1133. (doi:10.1016/S0006-3495(97)78145-1)

6 Simon, M. D. \& Geim, A. K. 2000 Diamagnetic levitation: flying frogs and floating magnets. J. Appl. Phys. 87, 62006204. (doi:10.1063/1.372654) 
7 Beaugnon, E., Fabregue, D., Billy, D., Nappa, J. \& Tournier, R. 2001 Dynamics of magnetically levitated droplets. Physica B 294, 715-720. (doi:10.1016/S09214526(00)00750-X)

8 Liu, Y., Zhu, D-M., Strayer, D. M. \& Israelsson, U. E. 2010 Magnetic levitation of large water droplets and mice. Adv. Space Res. 45, 208-213. (doi:10.1016/j.asr.2009.08.033)

9 Guevorkian, K. \& Valles, J. M. 2006 Swimming Paramecium in magnetically simulated enhanced, reduced, and inverted gravity environments. Proc. Natl Acad. Sci. USA 103, 13 051-13 056. (doi:10.1073/pnas.0601839103)

10 Coleman, C. B., Gonzalez-Villalobos, R. A., Allen, P. L., Johanson, K., Guevorkian, K., Valles, J. M. \& Hammond, T. G. 2007 Diamagnetic levitation changes growth, cell cycle, and gene expression of Saccharomyces cerevisiae. Biotechnol. Bioeng. 98, 854-863. (doi:10.1002/bit.21526)

11 Babbick, M., Dijkstra, C., Larkin, O. J., Anthony, P., Davey, M. R., Power, J. B., Lowe, K. C., Cogoli-Greuter, M. \& Hampp, R. 2007 Expression of transcription factors after short term exposure of Arabidopsis thaliana cell cultures to hypergravity and simulated microgravity $(2-\mathrm{D} / 3-$ D clinorotation, magnetic levitation). Adv. Space Res. 39, 1182-1189. (doi:10.1016/j.asr.2007.01.001)

12 Wilson, J. W. et al. 2007 Space flight alters bacterial gene expression and virulence and reveals a role for global regulator Hfq. Proc. Natl Acad. Sci. USA 104, $16299-16304$. (doi:10.1073/pnas.0707155104)

13 Ikehata, M., Iwasaka, M., Miyakoshi, J., Ueno, S. \& Koana, T. 2003 Effects of intense magnetic fields on sedimentation pattern and gene expression profile in budding yeast. J. Appl. Phys. 93, 6724-6726. (doi:10.1063/1. 1556929)

14 Glade, N., Beaugnon, E. \& Tabony, J. 2006 Ground-based methods reproduce space-flight experiments and show that weak vibrations trigger microtubule self-organisation. Biophys. Chem. 121, 1-6. (doi:10.1016/j.bpc.2005.12.001)

15 Qian, A. S., Di Gao, X., Zhang, W., Tian, Z., Li, J., Hu, L., Yang, P., Yin, D. \& Shang, P. 2009 cDNA microarray reveals the alterations of cytoskeleton-related genes in osteoblast under high magneto-gravitational environment. Acta Biochim. Biophys. Sin. 41, 561-577. (doi:10.1093/ abbs/gmp041)

16 Hill, R. J. A. \& Eaves, L. 2008 Nonaxisymmetric shapes of a magnetically levitated and spinning water droplet. Phys. Rev. Lett. 101, 234501. (doi:10.1103/PhysRevLett.101. 234501)

17 Hill, R. J. A. \& Eaves, L. 2010 Vibrations of a diamagnetically levitated water droplet. Phys. Rev. E 81, 056312. (doi:10.1103/PhysRevE.81.056312)

18 Kubitschek, H. E. 1986 Increase in cell mass during the division cycle of E. coli B/rA. J. Bacteriol. 168, 613-618.

19 Tanimoto, Y., Fujiwara, M., Sueda, M., Inoue, K. \& Akita, M. 2005 Magnetic levitation of plastic chips: applications for magnetic susceptibility measurement and magnetic separation. Jpn. J. Appl. Phys. 44, 6801-6803. (doi:10.1143/JJAP.44.6801)

20 Denegre, J. M., Valles Jr, J. M., Lin, K., Jordan, W. B. \& Mowry, K. L. 1998 Cleavage planes in frog eggs are altered by strong magnetic fields. Proc. Natl Acad. Sci. USA 95, 14 729-14 732. (doi:10.1073/pnas.95.25.14729)

21 Valles Jr, J. M. 2002 Model of magnetic field-induced mitotic apparatus reorientation in frog eggs. Biophys. J. 82, 1260-1265. (doi:10.1016/S0006-3495(02)75482-9)

22 Hill, R. J. A., Sedman, V. L., Allen, S., Williams, P., Paoli, M., Adler-Abramovich, L., Gazit, E., Eaves, L. \& Tendler, S. J. B. 2007 Alignment of aromatic peptide tubes in strong magnetic fields. Adv. Mater. 19, 4474-4479. (doi:10.1002/adma.200700590)
23 Steiner, U. \& Ulrich, T. 1989 Magnetic field effects in chemical kinetics and related phenomena. Chem. Rev. 89, 51-147 and references therein. (doi:10.1021/ cr00091a003)

24 Valles Jr, J. M., Maris, H. J., Seidel, G. M., Tang, J. \& Yao, W. 2005 Magnetic levitation-based martian and lunar gravity simulator. Adv. Space Res. 36, 114-118. (doi:10.1016/j.asr.2005.01.081)

25 Atlung, T., Knudsen, K., Heerfordt, L. \& Brøndsted, L. 1997 Effects of sigma(S) and the transcriptional activator AppY on induction of the Escherichia coli hya and $c b d A B$ appA operons in response to carbon and phosphate starvation. J. Bacteriol. 179, 2141-2146.

26 Cotter, P. A. \& Gunsalus, R. P. 1992 Contribution of the fnr and $\operatorname{arc} A$ gene-products in coordinate regulation of cytochrome-o and cytochrome-d oxidase (cyoABCDE and $c y d A B)$ genes in Escherichia coli. FEMS Microbiol. Lett. 91, 31-36. (doi:10.1111/j.1574-6968.1992. tb05179.x)

27 Ueno, S. \& Iwasaka, M. 1994 Properties of diamagnetic fluid in high gradient magnetic fields. J. Appl. Phys. 75, 7177-7179. (doi:10.1063/1.356686)

28 Hirota, N., Ikezoe, Y., Uetake, H., Nakagawa, J. \& Kitazawa, K. 2000 Magnetic field effect on the kinetics of oxygen dissolution into water. Mater. Trans. JIM 41, 976-980.

29 Kishioka, S., Yamada, A. \& Aogaki, R. 2000 Analysis of gas dissociation rate into liquid phase under magnetic field gradient. Phys. Chem. Chem. Phys. 2, 4179-4183. (doi:10.1039/b003472j)

30 Kitazawa, K., Hirota, N., Ikezoe, Y., Uetake, H., Kaihatsu, T. \& Takayama, T. 2002 Magneto-convection processes observed in non-magnetic liquid-gas system. Riken Rev. 44, 156.

31 Iwasaka, M., Yamamoto, K., Ando, J. \& Ueno, S. 2003 Verification of magnetic field gradient effects on medium convection and cell adhesion. J. Appl. Phys. 93, 67156717. (doi:10.1063/1.1558632)

32 Peng, Z. M., Wang, J., Huang, Y. J. \& Chen, Q. W. 2004 Magnetic field-induced increasing of the reaction rates controlled by the diffusion of paramagnetic gases. Chem. Eng. Technol. 27, 1273-1276. (doi:10.1002/ceat. 200402139)

33 Ikezoe, Y., Hirota, N., Nakagawa, J. \& Kitazawa, K. 1998 Making water levitate. Nature 393, 749-750. (doi:10. 1038/31619)

34 Catherall, A. T., Eaves, L., King, P. J. \& Booth, S. R. 2003 Magnetic levitation: floating gold in cryogenic oxygen. Nature 422, 579. (doi:10.1038/422579a)

35 Catherall, A. T., Lopez-Alcaraz, P., Benedict, K. A., King, P. J. \& Eaves, L. 2005 Cryogenically enhanced magnetoArchimedes levitation. New J. Phys. 7, 118-128. (doi:10. 1088/1367-2630/7/1/118)

36 Lopez-Alcaraz, P., Catherall, A. T., Hill, R. J. A., Leaper, M. C., Swift, M. R. \& King, P. J. 2007 Magneto-vibratory separation of glass and bronze granular mixtures immersed in a paramagnetic fluid. Eur. Phys. J. E 24, 145-156. (doi:10.1140/epje/i2007-10225-1)

37 Ueno, S. 1989 Quenching of flames by magnetic fields. J. Appl. Phys. 65, 1243-1245. (doi:10.1063/1.343016)

38 Aoyagi, S., Yano, A., Yanagida, Y., Tanihira, E., Tagawa, A. \& Iimoto, M. 2006 Control of chemical reaction involving dissolved oxygen using magnetic field gradient. Chem. Phys. 331, 137-141. (doi:10.1016/j.chemphys. 2006.10.006)

39 Poodt, P. W. G., Heijna, M. C. R., Tsukamoto, K., de Grip, W. J., Christianen, P. C. M., Maan, J. C., van Enckevort, W. J. P. \& Vlieg, E. 2005 Suppression of 
convection using gradient magnetic fields during crystal growth of $\mathrm{NiSO}_{4} \cdot 6 \mathrm{H}_{2} \mathrm{O}$. Appl. Phys. Lett. 87, 214105. (doi:10.1063/1.2133894)

40 Heijna, M. C. R., Poodt, P. W. G., Tsukamoto, K., de Grip, W. J., Christianen, P. C. M., Maan, J. C., Hendrix, J. L. A., van Enckevort, W. J. P. \& Vlieg, E. 2007 Magnetically controlled gravity for protein crystal growth. Appl. Phys. Lett. 90, 264105. (doi:10.1063/1.2752718)

41 Weast, R. 1972 Handbook of chemistry and physics, 53rd edn. Cleveland, OH: CRC Press.

42 Faber, T. E. 1995 Fluid dynamics for physicists. Cambridge, UK: Cambridge University Press.

43 Wilke, C. R. \& Chang, P. 1955 Correlation of diffusion coefficients in dilute solutions. AIChE J. 1, 264-270. (doi:10.1002/aic.690010222)

44 Poodt, P. W. G., Heijna, M. C. R., Christianen, P. C. M., van Enckevort, W. J. P., de Grip, W. J., Tsukamoto, K., Maan, J. C. \& Vlieg, E. 2006 Using gradient magnetic fields to suppress convection during crystal growth. Cryst. Growth Des. 6, 2275-2280. (doi:10.1021/cg0600546)

45 Nakamura, H., Takayama, T., Uetake, H., Hirota, N. \& Kitazawa, K. 2005 Magnetically controlled convection in a diamagnetic fluid. Phys. Rev. Lett. 94, 144501. (doi:10. 1103/PhysRevLett.94.144501)
46 Marrero, T. R. \& Mason, E. A. 1972 Gaseous diffusion coefficients. J. Phys. Chem. Ref. Data 1, 3-118. (doi:10. 1063/1.3253094)

47 Benson, B. B. \& Krause Jr, D. 1980 The concentration and isotopic fractionation of gases dissolved in freshwater in equilibrium with the atmosphere. 1. Oxygen. Limnol. Oceanogr. 25, 662-671. (doi:10.4319/lo.1980.25.4.0662)

48 Chen, J., Tannahill, A. L. \& Shuler, M. L. 1985 Design of a system for the control of low dissolved oxygen concentrations: critical oxygen concentrations for Azobacter vinelandii and Escherichia coli. Biotechnol. Bioeng. 27, 151-155. (doi:10.1002/bit.260270208)

49 Guerin, T. F., Mondido, M., McClenn, B. \& Peasley, B. 2001 Application of resazurin for estimating abundance of contaminant-degrading organisms. Lett. Appl. Microbiol. 32, 340-345. (doi:10.1046/j.1472-765X.2001. 00916.x)

50 Bovallius, A. \& Zacharias, B. 1971 Variations in the metal content of some commercial media and their effect on microbial growth. Appl. Microbiol. 22, 260-262.

51 Beuls, E., Van Houdt, R., Leys, N., Dijkstra, C. E., Larkin, O. J. \& Mahillon, J. 2009 Bacillus thuringiensis conjugation in simulated microgravity. Astrobiology 9, 797805. (doi:10.1089/ast.2009.0383) 